\title{
Images in clinical medicine: gouty arthritis with osteomyelitis
}

\author{
Rosa Scipioni, ${ }^{1}$ Luciano Frate, ${ }^{2}$ Valentino Di Tomasso, ${ }^{1}$ Michele Saltarelli, ${ }^{1}$ Francesco Carubbi, ${ }^{3}$ Marco Petrarca ${ }^{4}$ \\ ${ }^{1}$ Division of Internal Medicine, Castel Di Sangro Hospital, Castel Di Sangro (AQ); ${ }^{2}$ Division of Orthopaedic, Castel Di Sangro \\ Hospital, Castel Di Sangro (AQ); ${ }^{3}$ Rheumatology Unit, Department of Biotechnological and Applied Clinical Science, School \\ of Medicine, University of L'Aquila, L'Aquila; ${ }^{4}$ Division of Internal Medicine, Giuseppe Mazzini Hospital, Teramo, Italy
}

\begin{abstract}
Gout is one of the most common inflammatory arthropathies, characterized by the deposition of monosodium urate crystals in the synovial membrane, articular cartilage and periarticular tissues and leading to inflammation. The natural history of articular gout is typically composed of four periods: asymptomatic hyperuricemia, episodes of acute attacks of gout (acute gouty arthritis) with asymptomatic intervals (intercritical gout), and chronic tophaceous gout. Tophi develop in 12-35\% of gouty patients without adequate control of uricemia. Initially, they do not cause significant complaints or function limitation of the nearby joints. However, if they become larger, joint instability and movement range limitation, joint function impairment and bone erosions and infection at the sites of their penetration can develop. We report a case of a poorly controlled polyarticular tophaceous gout complicated by osteomyelitis.
\end{abstract}

\section{Introduction}

Gout is the most common inflammatory arthritis in the Western countries, with a prevalence of $1-4 \%$ in the general population. In these areas, it occurs in $3-6 \%$ of men and $1-2 \%$ of women. Worldwide incidence of gout gradually increases due to poor dietary habits such as fast foods, lack of exercise, increased incidence of obesity and metabolic syndrome. ${ }^{1}$ It is associated with functional impairment and, for many, a diminished health-related quality of life (QoL).

Gout is caused by the deposition of monosodium urate (MSU) crystals in joints and soft tissues when

Correspondence: Rosa Scipioni, Division of Internal Medicine, Giuseppe Mazzini Hospital, Piazza Italia, 64100 Teramo, Italy. Tel.: +39.0861429545. E-mail: rosascip@gmail.com

Key words: Gout; tophi; osteomyelitis.

Contributions: RS, manuscript writing, search for reference; RS, FC, MS, VDT, LF, case management; RS, LF, acquisition of the images; MP, manuscript review.

Conflict of interest: the authors declare no conflict of interest.

Received for publication: 23 May 2018.

Revision received: 25 July 2018.

Accepted for publication: 27 July 2018.

This work is licensed under a Creative Commons Attribution NonCommercial 4.0 License (CC BY-NC 4.0).

CCopyright R. Scipioni et al., 2018

Licensee PAGEPress, Italy

Italian Journal of Medicine 2018; 12:270-272

doi:10.4081/itjm.2018.1047 serum uric acid (UA) concentrations rise above the normal threshold, defined as $6.8 \mathrm{mg} / \mathrm{dL}^{2}$

The acute form of the arthritis is characterized by sudden onset, pain, swelling, warmth and erythema. Chronic deposition of MSU crystals leads to increased frequency of acute attacks, progressive shortening of intercritical phase and development of tophi due to MSU deposition in soft tissue, bones and joints. A tophus is a mass formed of large amounts of accumulated MSU crystals, it is a manifestation of chronic and uncontrolled disease. The GOLD standard diagnostic test for gout is the identification of MSU crystals by polarized light microscopy in synovial fluid cells or in a tophus. Despite the improvement on knowledge of mechanisms of disease and effective treatments, gout is still often misdiagnosed and its management suboptimal. ${ }^{3}$

\section{Case Report}

An 80-year-old man was admitted to our Division of Internal Medicine because of a 3-day fever (up to $39^{\circ} \mathrm{C}$ ) associated with pain and swelling in his right hand. He had a long-standing history of poorly controlled polyarticular tophaceous gout, mainly in his hands with difficulty in moving the extremities; he regularly took allopurinol $300 \mathrm{mg}$ per day. There was no family history of gout, but personal history of high purine diet intake. He had been a tobacco smoker until ten years ago, and denied alcoholism.

He was noted to have multiple hard swellings, which developed over 10 years, progressively increasing in size.

On Admission physical examination revealed multiple large firm tophi over bilateral hands, feet, 
elbows, and knees with marked digital deformity. The second right finger was erythematous, swollen, painful, ulcerated and discharged white chalky material. There was a severe loss of digital flexion and extension (Figure 1).

He was febrile $\left(38^{\circ} \mathrm{C}\right)$. Thoracic, cardiac and neurological findings were negative.

Laboratory tests showed leukocytosis $\left(14.19 \times 10^{3}\right.$ cells $/ \mu \mathrm{L})$ and elevated inflammatory markers: erythrocyte sedimentation rate of $120 \mathrm{~mm} / \mathrm{h}, \mathrm{C}$ reactive protein of $168 \mathrm{mg} / \mathrm{dL}$ and ferritin of 1064 $\mathrm{ng} / \mathrm{mL}$. The renal function test and uricemia were normal: blood urea $40 \mathrm{mg} / \mathrm{dL}$, serum creatinine 1.0 $\mathrm{mg} / \mathrm{dL}$, uricemia $6 \mathrm{mg} / \mathrm{dL}$.

Radiographs of both hands demonstrated bilaterally marked soft tissue swelling with virtually complete osteolysis of the middle and distal phalanges of the second right finger (Figure 2).

Needle aspiration yielded a white viscous liquid, with numerous urate crystals identified on polarized light microscopy. The culture sample was positive for Staphylococcus aureus, while the blood cultures were negative. Abdominal computed tomography did not identify any UA stones.

A diagnosis of osteomyelitis in erosive tophaceous gout was made. He was treated with intravenous antibiotic (ceftriaxone $2 \mathrm{~g}$ for 14 days and ciprofloxacin $400 \mathrm{mg}$ bid for 21 days), and he underwent an orthopedic complete amputation of the right index (Figure 3). Surgery obviously relieved the pain; one week later the patient was afebrile, laboratory tests showed an improvement of inflammatory markers and he was discharged with long-term treatment with colchicine $1 \mathrm{mg}$ per day and febuxostat $80 \mathrm{mg}$ per day. No further complications were observed.

\section{Discussion}

This case illustrates the debilitating, chronic manifestation of poorly controlled gout and the difficulty of its management.

Gout is a chronic deforming polyarthropathy and one of the most frequent causes of disability. ${ }^{4}$ Patients with gout have $56 \%$ more work loss days than population controls, partially explained by an increased frequency of comorbidities. Absenteeism is strongly predicted by socioeconomic status, age, gender, and place of residence (urban $v s$ rural community)..$^{5}$ Gout is especially disabling when upper extremities are damaged. Of note, the involvement of the hands occurs mainly in women, by contrast the localization in the first metatarsophalangeal joint (podagra) is more frequently observed in the males. ${ }^{6}$

Although hyperuricemia is the main pathogenic defect in gout, many people with hyperuricemia do not develop gout or even form MSU crystals: in particular, only $5 \%$ of people with hyperuricemia above $9 \mathrm{mg} / \mathrm{dL}$ develop gout. Normal serum UA levels were found in $63.3 \%$ of patients with acute gout attack. Accordingly,

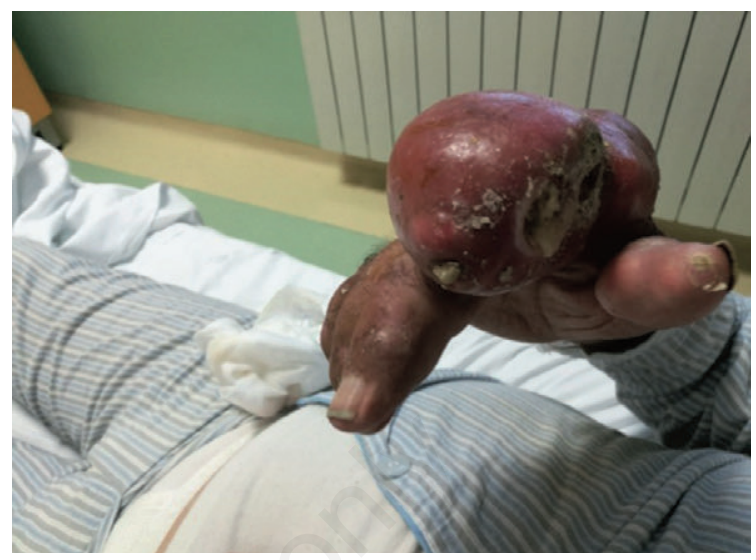

Figure 1. Erythematous, swollen, and ulcerated second right finger discharging white chalky material.

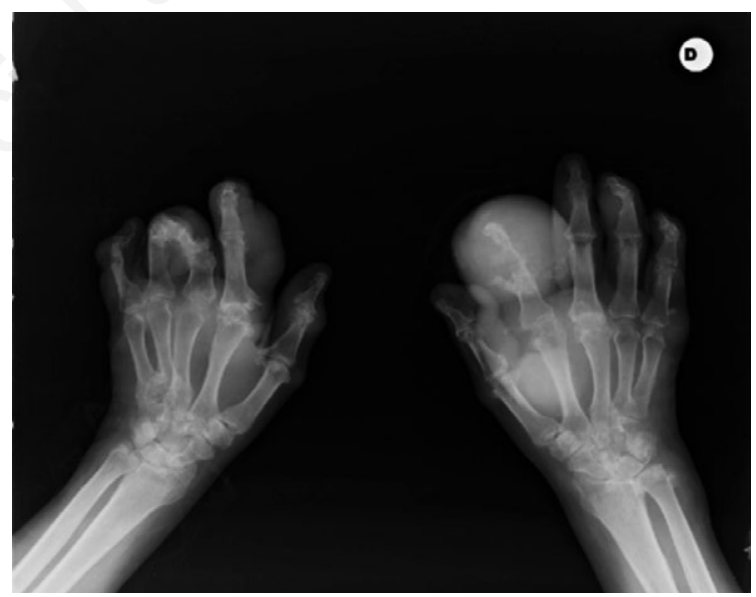

Figure 2. Radiography of both hands showing soft-tissue swelling with osteolysis of the middle and distal phalanges of the second right finger.

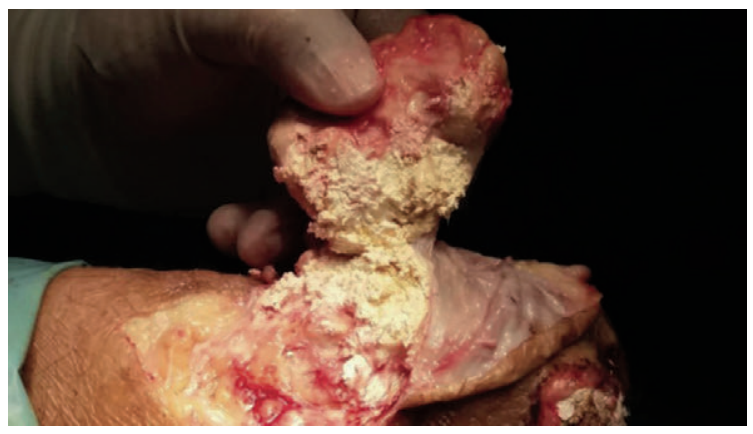

Figure 3. Intraoperative photo of the hand. 
it is thought that other factors such as genetic predisposition (e.g. SLC $2 A 9$ polymorphism) share in the incidence of gout. ${ }^{7,8}$

Gout results from the precipitation of MSU crystals in a joint space. Their deposition triggers immune activation with the release of several inflammatory cytokines and massive infiltration of neutrophils in the joint fluid and synovial membrane. ${ }^{9}$ Typically, after years of hyperuricemia, a foreign body granulomatous reaction will occur, leading to the development of the tophus. Tophi (i.e., subcutaneous nodules composed of MSU crystals in a matrix of lipids, protein, and mucopolysaccharides) are nodules of a palpable size generally placed near the joints and may be the initial clinical feature of gout, but are usually seen in longstanding undiagnosed or improperly treated gout. Over time, the joint space can be irreversibly damaged, leading to chronic pain and disability with grossly deformed joints. Although joint infections in tophaceous gout are a rare entity, they represent a severe complication, with significant morbidity and greatly impact on patients' QoL.

There are several possible mechanisms by which gout may increase the risk of septic arthritis. Deposition of MSU crystals can activate chondrocytes to release IL-1, inducible nitric oxide synthetase and matrix metalloproteinases, leading to cartilage destruction. ${ }^{10}$ The resulting joint damage can contribute to an increased risk of development of septic arthritis. ${ }^{11}$ Furthermore, the presence of subcutaneous tophi (especially in areas near the joint) predisposes patients to skin breakdown or ulceration. This may increase the risk of direct inoculation of bacteria, leading to septic arthritis. ${ }^{12}$ The incidence of septic arthritis in gouty patients is poor; a study on the United Kingdom population showed that gouty patients are 2.6 times more likely to be diagnosed with septic arthritis associated with antibiotic use or hospitalization. ${ }^{13}$

Lowering serum urate levels with xanthine oxidase inhibitors or uricosuric agents prevent acute flares and tophi development. The recommended target serum UA concentration is $<6 \mathrm{mg} / \mathrm{dL}(357 \mu \mathrm{mol} / \mathrm{L}){ }^{2}$ Although controversial, recommendations have been made to achieve a target serum urate level $<5 \mathrm{mg} / \mathrm{dL}$ in severe chronic gouty patients, as this concentration may be associated with greater depletion of synovial fluid crystals and a reduction in tophus size. ${ }^{14}$ Nevertheless, complete resolution of tophi can take years, and they will be present as a possible source of ulceration and infection for significant time. Surgical treatment is often considered to remove or decrease the size of subcutaneous tophi; its indications include: functional impairment, skin ulceration or tophaceous necrosis, local infection or septicemia, and nerve compression. ${ }^{15}$

\section{Conclusions}

In conclusion our report shows that gout and its longterm sequelae are often underestimated, and its poor control can lead to debilitating arthropathies. Although joint infections in tophaceous gout are a rare entity, they have a significant morbidity and greatly impact on patients' QoL. Further research should be aimed to identify the best medical, surgical and wound care regimens for the treatment of severe tophaceous gout.

\section{References}

1. Kuo CF, Grainge MJ, Zhang W, Doherty M. Global epidemiology of gout: prevalence, incidence and risk factors. Nat Rev Rheumatol 2015;11:649-62.

2. Neogi T. Clinical practice: gout. N Engl J Med 2011; 364:443-52.

3. Kuo CF, Grainge MJ, Mallen C, et al. Rising burden of gout in the UK but continuing suboptimal management: a nationwide population study. Ann Rheum Dis 2015;74:661-7.

4. Smith E, Hoy D, Cross M, et al. The global burden of gout: estimates from the Global Burden of Disease 2010 study. Ann Rheum Dis 2014;73:1470-6.

5. Sigurdardottir V, Drivelegka P, Svärd A, et al. Work disability in gout: a population-based case-control study. Ann Rheum Dis 2017;0:1-6.

6. De Souza AW, Fernandes V, Ferrari AJ. Female gout: clinical and laboratory features. J Rheumatol 2005; 32:2186-8.

7. Dalbeth N, Merriman TR, Stamp LK. Gout. Lancet 2016;388:2039-52.

8. Emmerson BT. The management of gout. N Engl J Med 1996;334:445-51.

9. Gonzalez EB. An update on the pathology and clinical management of gouty arthritis. Clin Rheumatol 2012; 3:13-21.

10. Choi HK, Mount DB, Reginato AM, et al. Pathogenesis of gout. Ann Int Med 2005;143:499-516.

11. Mathews CJ, Weston VC, Jones A, et al. Bacterial septic arthritis in adults. Lancet 2010;375:846-55.

12. Yu KH, Luo SF, Liou LB, et al. Concomitant septic and gouty arthritis - an analysis of 30 cases. Rheumatology 2003;42:1062-6.

13. Lim SY, Lu N, Choi HK. Septic arthritis in gout patients: a population-based cohort study. Rheumatology 2015; 54:2095-9.

14. Harrold L. New developments in gout. Curr Opin Rheumatol 2013;25:304-9.

15. Larmon WA, Kurtz JF. The surgical management of chronic tophaceous gout. J Bone Joint Surg Am 1958; 40A:743-72. 